Case Report

\title{
Subacute Presentation of Right Ventricular Perforation after Pacemaker Implantation
}

\author{
Sachi Koyama, MD, ${ }^{1,2}$ Keiichi Itatani, MD, $\mathrm{PhD},{ }^{1,2}$ Shunei Kyo, MD, $\mathrm{PhD},{ }^{1,2}$ \\ Rie Aoyama, $\mathrm{MD},{ }^{3}$ Taizo Ishiyama, $\mathrm{MD}, \mathrm{PhD},{ }^{3}$ Kazumasa Harada, $\mathrm{MD}, \mathrm{PhD},{ }^{3}$ and \\ Minoru Ono, $\mathrm{MD}, \mathrm{PhD}^{1,2}$
}

\begin{abstract}
We report an 87-year-old woman with right ventricular perforation due to a permanent pacemaker lead detected 4-days after implantation. The pacemaker lead was seen to perforate through the myocardium and pericardium and to reach the left pleural cavity. We removed the wire surgically by median sternotomy. The pericardial effusion was cloudy and yellowish, suggesting infection. However, no bacteria were detected by bacterial cultures of the pericardial effusion and pacing wire. The patient developed neither mediastinitis nor sepsis after the operation, and a new pacemaker was implanted safely one month later.
\end{abstract}

Keywords: permanent pacemaker, lead perforation, yellowish pericardial effusion

\section{Introduction}

With newer indications for the implantation of cardiac devices, increasing numbers of permanent pacemakers and defibrillators are being implanted each year. The incidence of complications resulting from the insertion of such devices ranges from $3 \%$ to $7 \%$. Lead perforation is a relatively rare complication with incidence of between $0.1 \%$ and $0.8 \% .^{1)}$ It typically occurs at the time of implantation or within 24 hours and rarely after the first 24 hours. ${ }^{2}$

Case reports have described subacute or late pacemaker lead perforation of the right atrium or right ventricle. This complication is both rare and important. Infec-

${ }^{I}$ Department of Cardiac Surgery, Tokyo Metropolitan Geriatric Hospital, Tokyo, Japan

${ }^{2}$ Department of Cardiac Surgery, the Graduate School of Medicine, The University of Tokyo, Tokyo, Japan

${ }^{3}$ Department of Cardiology, Tokyo Metropolitan Geriatric Hospital, Tokyo, Japan

Received: December 9, 2011; Accepted: January 31, 2012

Corresponding author: Keiichi Itatani, MD, PhD. Department of Cardiac Surgery, Tokyo Metropolitan Geriatric Hospital, 35-2, Sakae-cho, Itabashi-ku, Tokyo 173-0015, Japan

Email: keiichiitatani@gmail.com

(C)2012 The Editorial Committee of Annals of Thoracic and Cardiovascular Surgery. All rights reserved. tion, failure of the lead to pace or sense appropriately, erosions of the pulse generator, and subclavian vein thrombosis are well-recognized, delayed complications of cardiac devices implantation. ${ }^{2)}$ The predictors of post-implantation pericardial effusion, which serves as a marker of perforation, include concomitant use of another transvenous device, steroid use within 7 days, and old age. ${ }^{2)}$

We report a case of the patient who presented with a lead perforation 4 days after pacemaker implantation. The pericardial effusion was cloudy and yellow, suggesting infection of the implanted pacemaker. However, bacterial cultures were negative.

Case

An 87-year-old woman was followed at an outpatient psychiatric clinic for dementia. Five months prior to the present admission, her heart rate decreased, and she was diagnosed with sick sinus syndrome. A pacemaker was implanted from the left subclavian vein. An X-ray just after the operation showed the pacemaker wire to have been correctly placed. Slight redundancy of the pacing wire could be seen inside the superior vena cava (SVC). On the 2nd postoperative day (POD), she was delirious and moved her shoulders vigorously in circles throughout the night. On the 3rd POD, she had the symptom of 


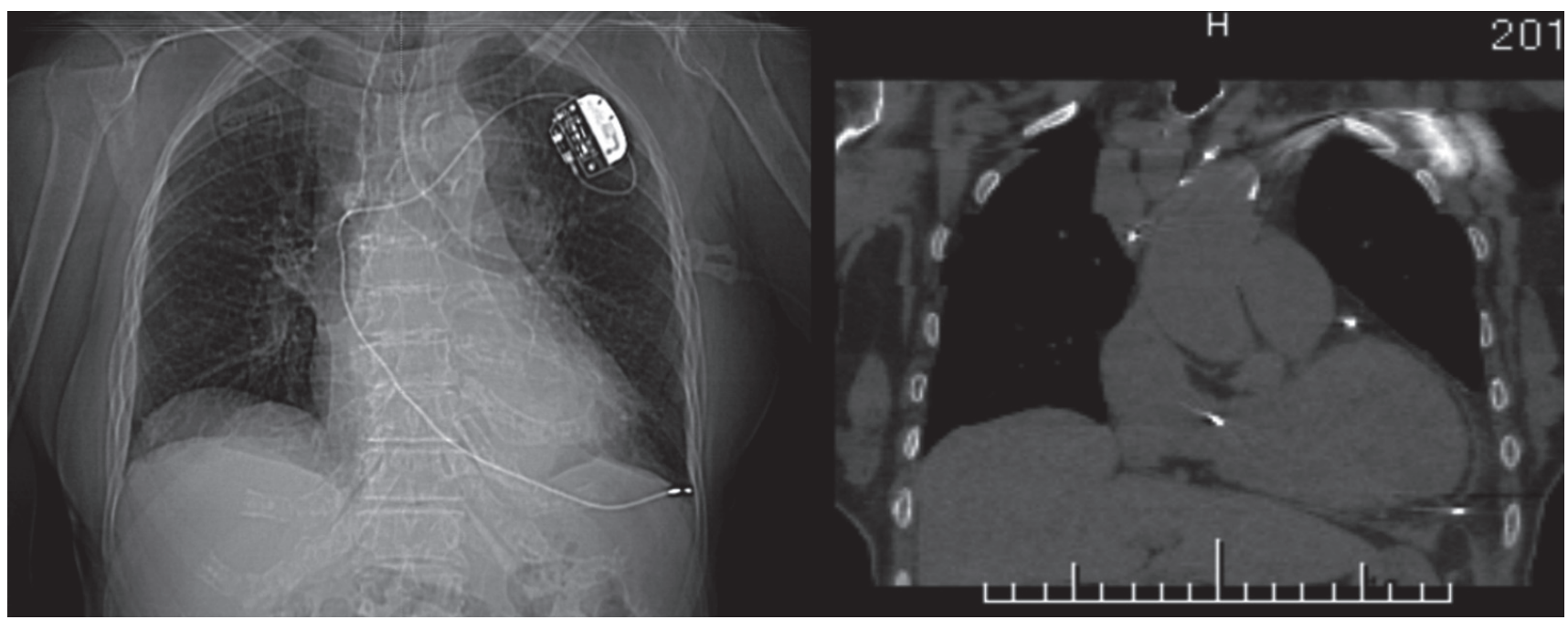

Fig. 1 A chest X-ray is on the left, a CT scan on the right. Both were obtained 4 days after pacemaker implantation. These images show penetration of the pacing wire through the right ventricle, reaching the left pleural cavity.

diaphragmatic twitching and electrocardiography showed pacing failure. On the 4th POD, she developed a sudden, high fever. Blood examinations showed a WBC of $6400 / \mu \mathrm{l}$, (segment was $81.3 \%$ ), CRP of $10.65 \mathrm{mg} / \mathrm{dl}$, and GOT/GPT of 189/437 IU/l. Computed tomography (CT) revealed pacemaker wire penetration through the right ventricle and into the left pleural cavity (Fig. 1). We elected to perform emergent surgery for removal of the pacemaker wire and to repair the right ventricle.

We employed the median sternotomy approach. Pericardiotomy revealed a cloudy, yellowish pericardial effusion. A pacing wire penetrated through the right ventricle, and there were no adhesions within the pericardial cavity intraoperatively (Fig. 2). We suspected an infection involving the implanted pacemaker or the subsequent explant procedure. Thus, we decided not to implant an epicardial permanent pacemaker at the time. We removed the pacing wire and the pacemaker generator, repaired the right ventricle with a single mattress suture, and washed the pericardial cavity using $2 \mathrm{~L}$ of saline. We then closed the sternum and skin.

After the operation, she remained afebrile. Her blood examinations revealed amelioration of the inflammatory response: WBC decreased to $4970 / \mu \mathrm{l}, \mathrm{CRP}$ to $0.25 \mathrm{mg} / \mathrm{dl}$, and GOT/GPT to 32/34 IU/1. Moreover, no bacteria were detected in cultures of the pericardial effusion and pacemaker wire. Thus, no reason was found for the cloudy yellowish appearance of the pericardial effusion. Fortunately, she did not develop mediastinitis.

One month after surgical repair, a new ventricular lead

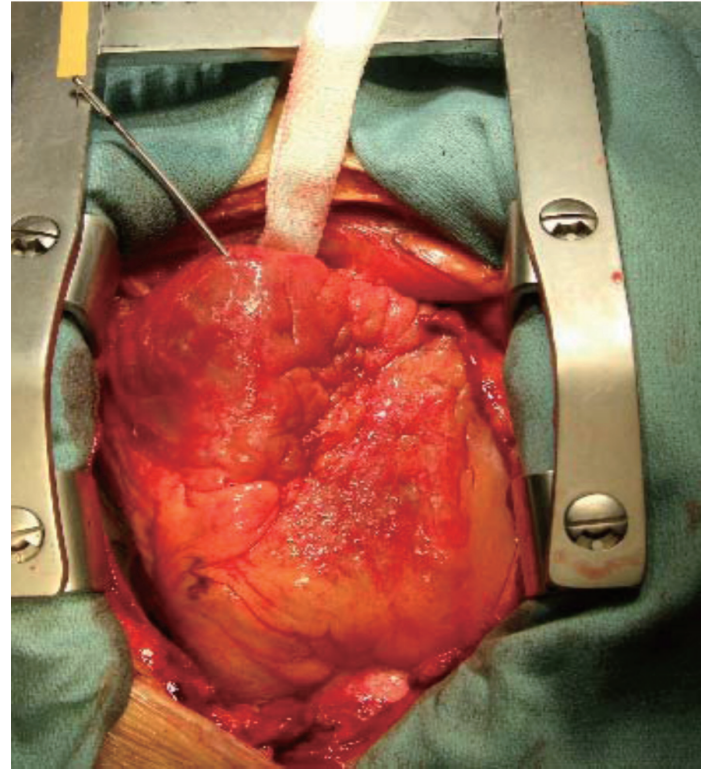

Fig. 2 Intra-operative view. The pacing wire penetrates through the right ventricle and epicardium, resulting in marked inflammation.

was placed at the right ventricular septum via a transvenous approach.

\section{Discussion}

Cardiac perforation is potentially one of the most serious complications of implanting a permanent pacemaker. ${ }^{3-5)}$ Lelorier noted that despite every precaution taken to avoid this complication, most operators, regard- 
less of skill and experience, will encounter a perforation. ${ }^{5)}$ The majority of perforations are reportedly due to manipulation of the pacing lead. ${ }^{4}$ Perforation of the right ventricle as a subacute or late complication after device implantation is rare. Routine chest radiography is not consistently diagnostic, and further image modalities such as CT and echocardiography are essential, combined with regular pacemaker interrogation. Moreover, the predictors of post-implantation pericardial effusion, which serves as a marker of perforation, are concomitant use of transvenous devices, steroid use within 7 days, and advanced age. $^{2)}$

In our case, the pacemaker wire was in the correct position on a chest X-ray obtained just after the operation. However, the pacemaker wire inside the SVC appeared to be too long. No other transvenous device or steroids had been used in this case. However, on chest X-ray the position of her innominate vein appeared to be anomalous and the wire insertion was thus slightly longer than usual. In addition, she had the risk factors of advanced age and dementia.

Perforation of the right ventricle could not be diagnosed solely on chest X-ray and blood examinations. CT established an accurate diagnosis of lead perforation. Moreover, she suffered a high fever and the pericardial effusion in the pericardium was cloudy and yellowish. However, no organisms were detected in any bacterial cultures. Thus, we concluded that she did not have an infection and explant surgery was successfully carried out without complications.

As in previous reports, clinical suspicion and a prompt response including surgical repair are important for avoiding cardiac tamponade. Nowadays, hospitalizations for pacemaker implantation are generally very brief, just a few days. Even after discharge, we must check the pacing wire frequently in the outpatient clinic to avoid overlooking the type of complication experienced by our present patient.

\section{Conclusion}

Subacute or late cardiac perforation after pacemaker implantation is a rare but possible complication. Inflammation is a potential cause, and just after the diagnosis, wire removal and careful ventricular repair are to be performed. We should continue to check this complication even after discharge.

\section{References}

1) Ramirez MF, Ching CK, Ho KL, et al. "The attack of the $52 \mathrm{~cm}$ lead": an unusual case of late cardiac perforation by a passive-fixation permanent pacemaker lead. Int J Cardiol 2007; 115: e5-7.

2) Haq SA, Heitner JF, Lee L, et al. Late presentation of a lead perforation as a complication of permanent pacemaker insertion. Angiology 2008; 59: 619-21.

3) Singhal S, Cooper JM, Cheung AT, et al. Images in cardiovascular medicine. Rib perforation from a right ventricular pacemaker lead. Circulation 2007; 115: e391-2.

4) Garcia-Bolao I, Teijeira R, Diaz-Dorronsoro I. Late fetal right ventricular perforation as complication of permanent pacing leads. Pacing Clin Electrophysiol 2001; 24: 1036-7.

5) Lelorier P. Accidents will happen (so be prepared). Heart Rhythm 2005; 2: 912-3. 